\title{
OPEN Machine learning models for screening carotid atherosclerosis in asymptomatic adults
}

\begin{abstract}
Jian $\mathrm{Yu}^{1,4}$, Yan Zhou ${ }^{2,3,4}$, Qiong Yang ${ }^{1}$, Xiaoling Liu ${ }^{1}$, Lili Huang ${ }^{1}$, Ping Yu ${ }^{1}$ \& Shuyuan Chu ${ }^{3 凶}$
Carotid atherosclerosis (CAS) is a risk factor for cardiovascular and cerebrovascular events, but duplex ultrasonography isn't recommended in routine screening for asymptomatic populations according to medical guidelines. We aim to develop machine learning models to screen CAS in asymptomatic adults. A total of $\mathbf{2 7 3 2}$ asymptomatic subjects for routine physical examination in our hospital were included in the study. We developed machine learning models to classify subjects with or without CAS using decision tree, random forest (RF), extreme gradient boosting (XGBoost), support vector machine (SVM) and multilayer perceptron (MLP) with 17 candidate features. The performance of models was assessed on the testing dataset. The model using MLP achieved the highest accuracy $(0.748)$, positive predictive value $(0.743), \mathrm{F} 1$ score $(0.742)$, area under receiver operating characteristic curve (AUC) (0.766) and Kappa score (0.445) among all classifiers. It's followed by models using XGBoost and SVM. In conclusion, the model using MLP is the best one to screen CAS in asymptomatic adults based on the results from routine physical examination, followed by using XGBoost and SVM. Those models may provide an effective and applicable method for physician and primary care doctors to screen asymptomatic CAS without risk factors in general population, and improve risk predictions and preventions of cardiovascular and cerebrovascular events in asymptomatic adults.
\end{abstract}

Carotid atherosclerosis (CAS) is a chronic disease with pathological thickening in common or internal carotid intima $^{1}$. It significantly increases the risk of ischemic stroke, coronary event and airflow limitation ${ }^{2-4}$. The global prevalence of CAS among people aged 30-90 years is estimated to be $27.6 \%$ in 2020 , namely more than one billion people suffered this disease ${ }^{5}$. In China, the prevalence of carotid plaques is about $31 \%$ among general population, and is $39 \%$ at the age of $60-69$ years $^{6}$. CAS is usually asymptomatic unless the patients suffered symptomatic ischemic stroke, transient ischemic attack, or amaurosis fugax ${ }^{1}$. Thus, CAS put a great health burden worldwide. If CAS in asymptomatic adults can be detected, it could improve risk predictions and preventions of cardiovascular and cerebrovascular events, particularly cardiovascular death, myocardial infarction and ischemic stroke ${ }^{7}$.

Carotid duplex ultrasonography is a noninvasive, safe and easily applicable diagnostic tool for detecting CAS, and has been widely used in CAS diagnosis ${ }^{8}$. However, carotid duplex ultrasonography is not recommended in routine screening for asymptomatic subjects who have no clinical manifestations or risk factors of atherosclerosis $^{9-11}$. Thus, we aim to develop classification model to screen the asymptomatic CAS based on data of routine physical examination from general population, which could help prevent cardiovascular and cerebrovascular events in asymptomatic population.

In recent years, machine learning has been widely used in medical study, and holds the promise to automatically diagnose heterogeneous diseases with high accuracy ${ }^{12}$. It's also successfully used in studies on CAS and cardiovascular disease ${ }^{13-15}$. In this study, we will develop models using decision tree, random forest (RF), extreme gradient boosting (XGBoost), support vector machine (SVM) and multilayer perceptron (MLP) based on the data from general population without symptoms of CAS. We'll assess the performance of those models and select good one. Those models will help to screen CAS in asymptomatic adults.

${ }^{1}$ Department of Endocrinology, Affiliated Hospital of Guilin Medical University, Guilin, Guangxi, China. ${ }^{2}$ Department of Respiratory and Critical Care Medicine, Affiliated Hospital of Guilin Medical University, Guilin, Guangxi, China. ${ }^{3}$ Laboratory of Respiratory Disease, Affiliated Hospital of Guilin Medical University, Guilin 541001, Guangxi, China. ${ }^{4}$ These authors contributed equally: Jian Yu and Yan Zhou. ${ }^{\circledR}$ email: emilyyuanchu@163.com 


\section{Results}

Subjects characteristics. A total of 2732 subjects were included in the study, among which 942 (34.5\%) subjects were diagnosed as CAS. Compared with Non-CAS group, CAS group was in older age (CAS group vs Non-CAS group: $56.3 \pm 7.4$ vs $49.4 \pm 6.8 \mathrm{yrs}$ ), and had higher blood pressure (systolic blood pressure (SP): $132 \pm 20$ vs $123 \pm 18 \mathrm{mmHg}$; diastolic blood pressure (DP): $80 \pm 12$ vs $76 \pm 12 \mathrm{mmHg}$ ), higher blood uric acid (UA) level (376.5 \pm 96.9 vs $352.7 \pm 93.8)$, higher homocysteine (HCY) level (13.22 \pm 5.86 vs $11.70 \pm 5.31 \mu \mathrm{mol} / \mathrm{L})$, and worse renal function (blood urea nitrogen (BUN): $5.1 \pm 1.5$ vs $4.7 \pm 1.2 \mathrm{mmol} / \mathrm{L}$; serum creatinine (Scr): $81.64 \pm 21.80 \mathrm{vs} 76.81 \pm 16.10 \mu \mathrm{mol} / \mathrm{L}$ ), (Table 1 ). Moreover, CAS group had a higher proportion of males and the subjects with nonalcoholic fatty liver disease (NAFLD), compared with Non-CAS group (Table 1).

Model performance. As Table 2 illustrated, the model using MLP showed the best performance among all classifiers with the highest accuracy (0.748), positive predictive value (PPV) (0.743), F1 score (0.742), area under receiver operating characteristic curve (AUC) (0.766) and Kappa score (0.445). The second-best performance was from models using XGBoost and SVM. They were showed very similar performance in testing data. The model using XGBoost showed a bit higher of F1 score (XGBoost vs SVM $=0.735$ vs 0.733 ), AUC ( 0.763 vs 0.757 ), and Kappa score $(0.429$ vs 0.413$)$ than using SVM. The model using RF showed worse performance than using SVM and XGBoost. And the worst performance was from model using decision tree. The receiver operating characteristic curves (ROCs) of all models were showed in Fig. 1.

Important features from the models. In this study, classifiers using decision tree, RF, XGBoost and SVM could show the important features in the model. In classifier using decision tree, age was the most important feature, followed with Dp, Sp and HCY (Fig. 2). Since the maxed depth was three in the decision tree from grid-search and tenfold cross-validation, those four features were selected as most important from the model. In classifier using RF, all features could be ranked based on the importance in the model. As showed in Fig. 3, the most important feature was age, followed by fasting plasma glucose (FPG), Sp, HCY, UA, total cholesterol (TC), Dp, BUN, serum aspartate aminotransferase (AST), low-density lipoprotein cholesterol (LDL-C), high-density lipoprotein cholesterol (HDL-C) and others. In classifier using XGBoost, the features selected from model were age, Dp, HDL-C, HCY, Sp, FPG and gender (Fig. 4). In classifier using SVM, the features could be selected using the support vector machine recursive feature elimination (SVM-RFE) algorithm ${ }^{16}$, which could optimize the performance of the classifier. The selected features were age, gender, Sp, Dp, TC, HDL-C, AST and ALT, which were in the same importance without further ranked in the SVM-RFE.

\section{Discussion}

In this study, we developed models using decision tree, RF, XGBoot, SVM and MLP to classify subjects with CAS from asymptomatic adults based on data of routine physical examination. All models were assessed by accuracy, PPV, F1 score and AUC. The best performance was from model using MLP, followed by XGBoost and SVM.

Although carotid duplex ultrasonography is used in CAS diagnosis, there's no evidence to support the routine ultrasonography screening among general subjects without symptoms or risk factors ${ }^{9-11}$. However, CAS is usually asymptomatic until it leads to serious outcomes, such as cardiovascular and cerebrovascular accident ${ }^{1}$. Considering the high prevalence of $\mathrm{CAS}^{5,6}$, it's necessary to propose an effective, noninvasive and convenient method for screening asymptomatic subjects. Machine learning model from our study is such a method, which could improve risk predictions and preventions of cardiovascular and cerebrovascular events in asymptomatic adults. That may have important clinical and public health implications.

In our study, we developed models using decision tree, RF, XGBoost, SVM and MLP. MLP is artificial neural network, which usually showed good performance (i.e. high accuracy, PPV and AUC) among machine learning models. However, MLP can't show the important features in the model, or can't be explained ${ }^{17-19}$. In contrast, decision tree could show the features with visualization and can be explained. In our study, criterion = entropy was selected with grid-search in the decision tree, which means C4.5 tree was developed. RF and XGBoost integrate many decision trees to promote the efficiency and accuracy of a signal tree ${ }^{18}$. Moreover, SVM is also a strong classifier in medical research, which could show important features in the model ${ }^{20}$. Thus, in addition to MLP, we developed models using decision tree, RF, XGBoost and SVM.

For all models in our study, the model using MLP showed the best performance with highest accuracy, PPV, F1 score, AUC and Kappa score. MLP is a neural network with one or more layer of neurons linked together through weighted synapses, in which learning takes place through the backpropagation of the network output error and updating the weights ${ }^{21}$. In our study, the single-hidden layer MLP (hidden_layer_sizes $=(100$,$) ) showed$ the best performance. Although MLP could include multiple-hidden layer, a model with single-hidden layer with enough nodes and right set of weights can learn any function and get the best results, which moreover could run faster than that with multiple-hidden layer ${ }^{22}$. Followed MLP, the model using SVM showed good performance. SVM is an effective approach for classification by using linear functions or special nonlinear functions, namely kernels, to transform the input space into a multidimensional space ${ }^{23}$. Thus, the model using SVM is a good classifier $^{18}$, which was confirmed in our study.

In addition, models using XGBoost and RF performed better than decision tree in our study, since both XGBoost and RF integrate decision trees to promote performance of signal tree model ${ }^{15,18}$. Moreover, the performance of model with XGBoost was similar with that using SVM, and was better than that based on RF in our study. For the principle of algorithm, XGBoost is a library based on the gradient increase framework ${ }^{24-26}$. In contrast, $\mathrm{RF}$ is a combination of multiple tree predictions, in which each tree depends on the values of a randomly sampled independent vector ${ }^{27}$. And all trees have the same distribution in the forest ${ }^{27}$. Thus, the model 


\begin{tabular}{|l|l|l|r|}
\hline Variables & $\begin{array}{l}\text { CAS group } \\
(\mathbf{n = 9 4 2})\end{array}$ & $\begin{array}{l}\text { Non-CAS group } \\
(\mathbf{n = 1 7 9 0})\end{array}$ & P values \\
\hline Gender $(\mathrm{male})$ & $686(72.8 \%)$ & $1058(59.1 \%)$ & $<0.001$ \\
\hline Age $($ years $)$ & $56.3 \pm 7.4$ & $49.4 \pm 6.8$ & $<0.001$ \\
\hline BMI $\left(\mathrm{kg} / \mathrm{m}^{2}\right)$ & $25.1 \pm 3.0$ & $24.7 \pm 3.1$ & 0.003 \\
\hline SP $(\mathrm{mmHg})$ & $132 \pm 20$ & $123 \pm 18$ & $<0.001$ \\
\hline DP $(\mathrm{mmHg})$ & $80 \pm 12$ & $76 \pm 12$ & $<0.001$ \\
\hline AST $(\mathrm{U} / \mathrm{L})$ & $20.87 \pm 7.86$ & $20.22 \pm 7.27$ & 0.031 \\
\hline ALT $(\mathrm{U} / \mathrm{L})$ & $22.94 \pm 12.53$ & $22.35 \pm 13.38$ & 0.269 \\
\hline BUN $(\mathrm{mmol} / \mathrm{L})$ & $5.1 \pm 1.5$ & $4.7 \pm 1.2$ & $<0.001$ \\
\hline Scr $(\mu \mathrm{mol} / \mathrm{L})$ & $81.64 \pm 21.80$ & $76.81 \pm 16.10$ & $<0.001$ \\
\hline TG $(\mathrm{mmol} / \mathrm{L})$ & $1.93 \pm 1.87$ & $1.72 \pm 1.48$ & 0.003 \\
\hline TC $(\mathrm{mmol} / \mathrm{L})$ & $4.89 \pm 0.87$ & $4.74 \pm 0.83$ & $<0.001$ \\
\hline LDL-C $(\mathrm{mmol} / \mathrm{L})$ & $3.29 \pm 0.81$ & $3.14 \pm 0.81$ & $<0.001$ \\
\hline HDL-C $(\mathrm{mmol} / \mathrm{L})$ & $1.24 \pm 0.35$ & $1.29 \pm 0.33$ & $<0.001$ \\
\hline UA $(\mu \mathrm{mol} / \mathrm{L})$ & $376.5 \pm 96.9$ & $352.7 \pm 93.8$ & $<0.001$ \\
\hline HCY $(\mu \mathrm{mol} / \mathrm{L})$ & $13.22 \pm 5.86$ & $11.70 \pm 5.31$ & $<0.001$ \\
\hline FPG $(\mathrm{mmol} / \mathrm{L})$ & $5.88 \pm 1.68$ & $5.47 \pm 1.21$ & $<0.001$ \\
\hline NAFID $(\mathrm{Yes})$ & $285(30.3 \%)$ & $417(23.3 \%)$ & $<0.001$ \\
\hline
\end{tabular}

Table 1. Subjects characteristics in CAS group and non-CAS group. CAS, carotid atherosclerosis; BMI, body mass index; SP, systolic blood pressure; DP, diastolic blood pressure; AST, serum aspartate aminotransferase; ALT, serum alanine aminotransferase; BUN, blood urea nitrogen; Scr, serum creatinine; TG, triglyceride; TC, total cholesterol; LDL-C, low-density lipoprotein cholesterol; HDL-C, high-density lipoprotein cholesterol; UA, blood uric acid; HCY, homocysteine; FPG, fasting plasma glucose; NAFLD, nonalcoholic fatty liver disease.

using XGBoost could promote performance more efficient than the one using RF, and then perform better than RF model.

Among all models in our study, models using decision tree, RF, XGBoost and SVM could show important features. Our results showed that age, Sp, Dp, HCY level and HDL-C level were most important in all those four models, followed by gender, TC level and FPG level. Our findings were in consistent with previous studies, in which older age, gender, high Sp, hypertension, high TC level and high FPG levle were independently related to the risk of CAS ${ }^{5,28-30}$. High HCY level was also associated with the progression of CAS ${ }^{31}$. And high HDL-C level was a protective factor for CAS reported in a study with Chinese population ${ }^{30}$. Thus, models using decision tree, RF, XGBoost and SVM in our study suggested that age, Sp, Dp, HCY level, HDL-C level, gender, TC level and FPG level should be important in screening CAS in general and asymptomatic adults.

We acknowledged the limitation in our study that smoking history was not included in candidate features for developing models. It's widely accepted that smoking is a risk factor for CAS ${ }^{5,28-30}$. However, no record of smoking history in our study. That may reduce the performance of our models, in which the AUC, accuracy, PPV and F1 score were less than 0.8, even in the best model using MLP. Thus, if smoking history was included in models, the performance should be improved.

In conclusion, it could create classification models using machine learning based on the results of routine physical examination. Those classifiers could screen CAS in asymptomatic adults without redundant examination. The model using MLP is the best one, followed by using XGBoost and SVM. Those models may provide an effective and applicable method for physician and primary care doctors to screen asymptomatic CAS without risk factors in general population, which could improve risk predictions and preventions of cardiovascular and cerebrovascular events in asymptomatic adults.

\section{Subjects and methods}

Study population. The subjects were recruited into this study from general people who took routine physical examination in the Center of Health Examination, Affiliated Hospital of Guilin Medical University, from July to October in 2017. All laboratory testing and quality control were carried out by the laboratory analysis center of our hospital. The study protocol was approved by the Research Ethics Committee of the Affiliated Hospital of Guilin Medical University, and conformed to the declaration of Helsinki. Written informed consent was obtained from each subject.

The inclusion criteria for subjects in the study were as following: (1) male or female; (2) age $\geq 20$ years; (3) subjects underwent carotid duplex ultrasonography; (4) subjects received blood testing on liver function, renal function, triglyceride (TG), TC, lipoprotein, HCY and FPG.

The exclusion criteria for subjects were: (1) had clinical manifestations of CAS including ipsilateral amaurosis fugax, retinal infarction, symptomatic ischemic stroke, or transient ischemic attack; (2) had a history of coronary atherosclerosis or coronary heart disease; (3) had autoimmune disorders; (4) had psychiatric disorders; or (5) had malignant tumor. 


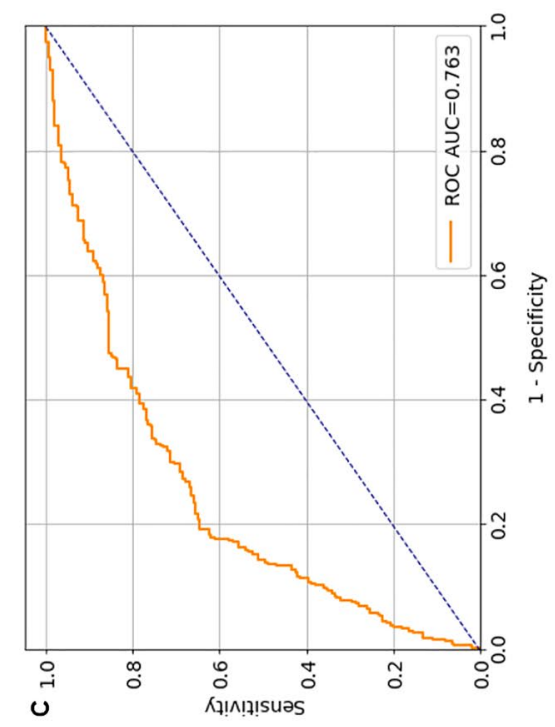

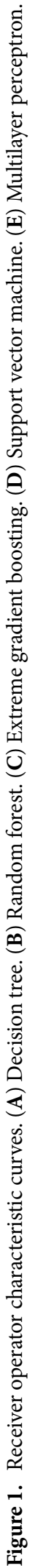




\begin{tabular}{|l|l|l|l|l|l|}
\hline Model & Accuracy & PPV & F1 score & Kappa score & AUC (95\% CI $)$ \\
\hline MLP & 0.748 & 0.743 & 0.742 & 0.445 & $0.766(0.754-0.769)$ \\
\hline XGBoost & 0.741 & 0.736 & 0.735 & 0.429 & $0.763(0.724-0.764)$ \\
\hline SVM & 0.744 & 0.739 & 0.733 & 0.413 & $0.757(0.718-0.757)$ \\
\hline Random forest & 0.730 & 0.724 & 0.722 & 0.401 & $0.752(0.734-0.766)$ \\
\hline Decision tree & 0.726 & 0.723 & 0.706 & 0.354 & $0.741(0.699-0.749)$ \\
\hline
\end{tabular}

Table 2. Model performance in testing data according to ranking. PPV, positive predictive value; AUC, area under curve; CI, confidence interval; MLP, multilayer perceptron; SVM, support vector machine; XGBoost, extreme gradient boosting.

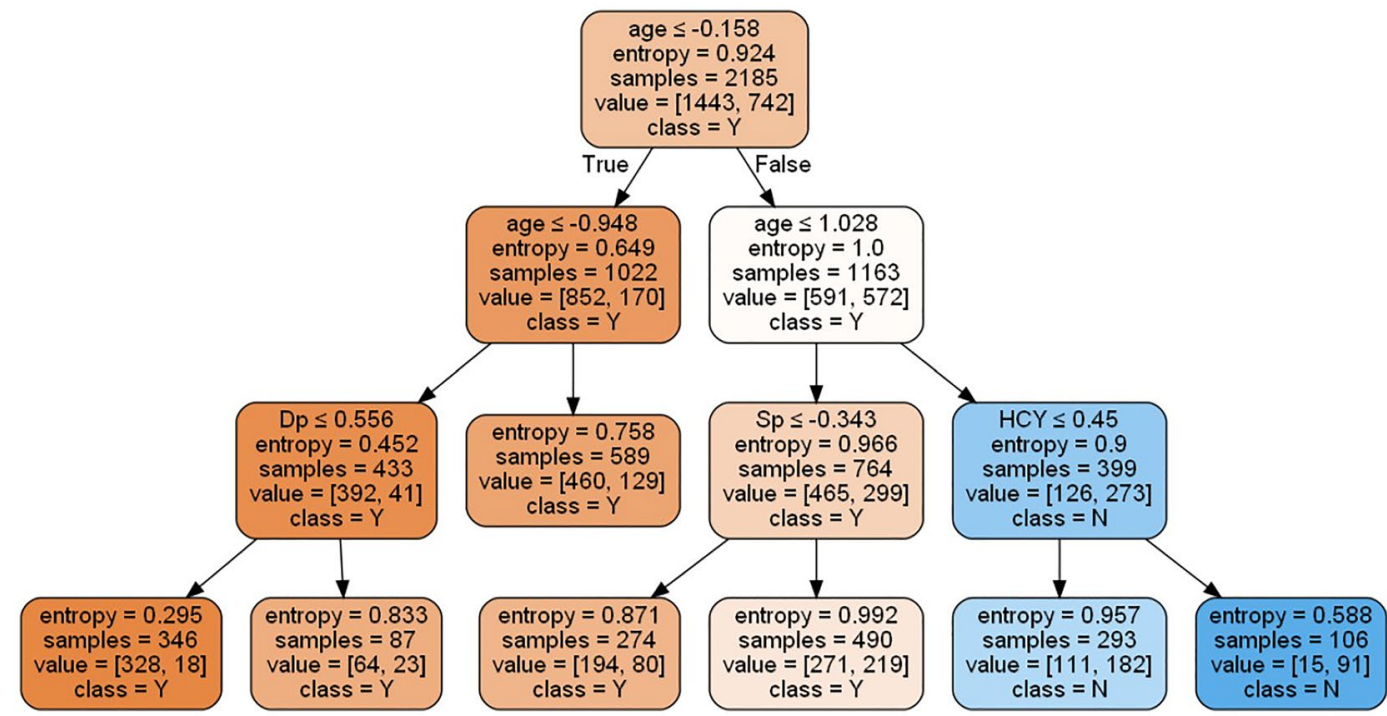

Figure 2. Decision tree. DP, diastolic blood pressure; SP, systolic blood pressure; HCY, homocysteine; $\mathrm{Y}=$ Yes; $\mathrm{N}=$ No.

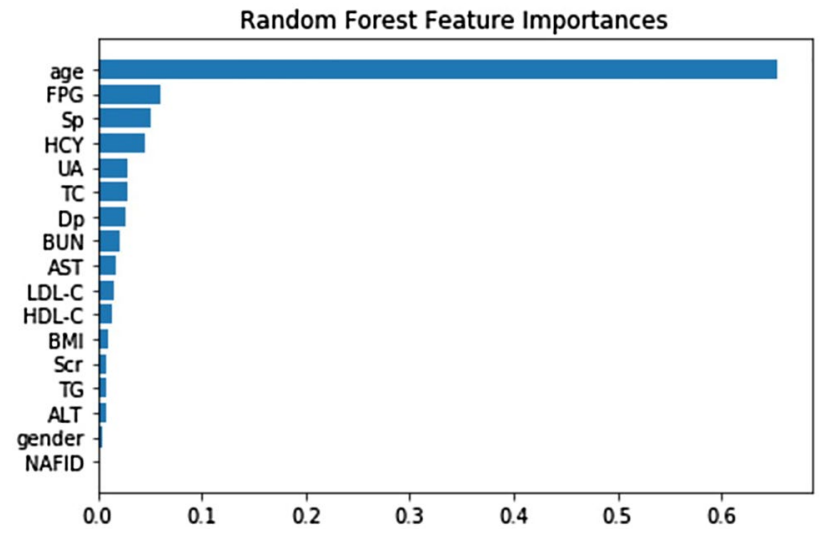

Figure 3. Features importance in random forest model. FPG, fasting plasma glucose; SP, systolic blood pressure; HCY, homocysteine; UA, blood uric acid; TC, total cholesterol; DP, diastolic blood pressure; BUN, blood urea nitrogen; AST, serum aspartate aminotransferase; LDL-C, low-density lipoprotein cholesterol; HDLC, high-density lipoprotein cholesterol; BMI, body mass index; Scr, serum creatinine; TG, triglyceride; ALT, serum alanine aminotransferase; NAFLD, nonalcoholic fatty liver disease. 


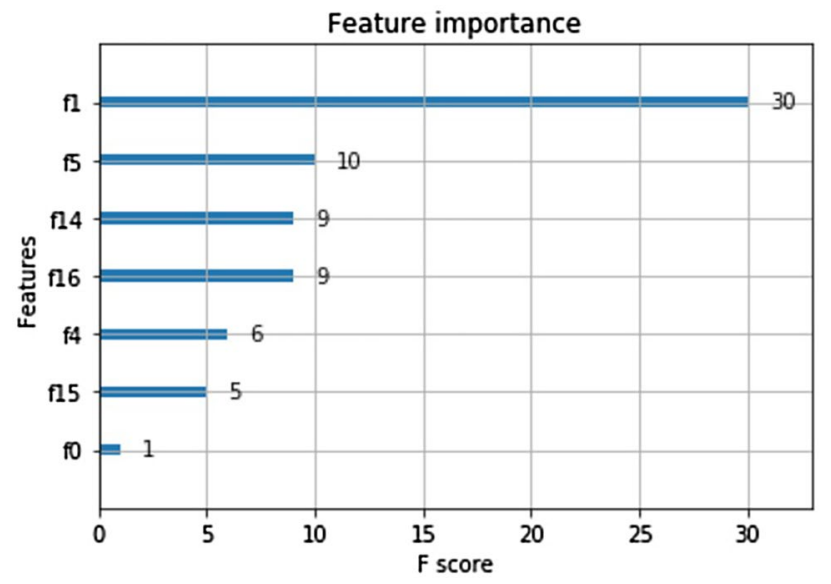

Figure 4. Features importance in XGBoost model. f1: age; f5: Dp; f14: HDL-C; f16: HCY; f4: Sp; f15: FPG; f0: gender.

Diagnostic criteria. The CAS was determined if carotid intima-media thickness (CIMT) $\geq 1 \mathrm{~mm}$ with or without atherosclerotic plaque ${ }^{32}$. The CIMT was automatically measured on the far wall of the left common carotid artery $10 \mathrm{~mm}$ proximal to the carotid bifurcation at end-diastole ${ }^{32,33}$ using color Doppler ultrasound with a 7.5-MHZ probe (DC-6 Expert, Mindray, Shenzhen, China) by exporters with at least 5 years' experience. NAFLD was diagnosed when there was evidence of hepatic steatosis by color Doppler ultrasound with a 3.5MHZ probe (DC-6 Expert, Mindray, Shenzhen, China) and there was no history of significant alcohol consumption, use of steatogenic medication, viral hepatitis, or hereditary disorders ${ }^{34}$.

Candidate features to classify subjects. The candidate features were collected from the electronic medical record. They were age, gender, NAFLD (Yes/No), BMI (BMI = weight/height ${ }^{2}$ ), SP, DP, UA, BUN, Scr, AST, serum alanine aminotransferase (ALT), TG, TC, LDL-C, HDL-C, FPG, and HCY.

Machine learning classifiers. In each group of subjects, $80 \%$ were randomly selected (training sample), who were used to develop the model. The remaining 20\% (testing sample) served to test the model. The training data were standardized using $\mathrm{z}$-score transformation, and the testing data were also transformed using the same parameters as those from the training data.

The models were developed using Python3.7.6 programming language (http://www.python.org), scikit-learn 22.2 library (https://scikit-learn.org/stable/). We developed models to classify subjects with CAS or without using decision tree, RF, XGBoost, SVM and MLP. The grid-search and tenfold cross-validation were used to estimate hyper parameters with training dataset. When several parameter combinations were optimal and the choice affected the efficiency of the model, we choose parameter combination which led to the highest efficiency. The hyper parameters of model using decision tree were $\max \_$depth $=3$, max_leaf_nodes $=7$ and criterion $=$ entropy; RF were n_estimators $=10$, max_depth $=5$, min_samples_split $=76$, min_sample_leaf $=35$, max_features $=7$; XGBoost were max_depth $=3 ; \mathrm{n} \_$estimators $=100$; learning rate $=0.1 ; \mathrm{SVM}$ were kernal $=\mathrm{rbf} ; \mathrm{C}=1.0$; and MLP were hidden_layer_sizes $=(100)$, activation $=$ logistic, solver $=$ adam, alpha $=0.1$, max_iter $=100$ (Supplement Table 1).

The performance of classifiers was assessed on the testing dataset, which was not used during the training step. The performance of models was assessed using accuracy, PPV, F1 score, AUC and Kappa score.

Statistical analysis. The continuous variables between case and control groups were analyzed with independent-samples t-test, and the categorical data were compared with Chi-square test. P-values $<0.05$ were considered to be statistically significant. Data were analyzed using SAS 9.4 (SAS Institute Inc., Cary, NC, USA).

Received: 30 April 2021; Accepted: 25 October 2021

Published online: 15 November 2021

\section{References}

1. Thapar, A., Jenkins, I. H., Mehta, A. \& Davies, A. H. Diagnosis and management of carotid atherosclerosis. BMJ 346, f1485 (2013).

2. Borné, Y. et al. Cadmium, carotid atherosclerosis, and incidence of ischemic stroke. J. Am. Heart Assoc. 6(12), e006415 (2017).

3. Sirimarco, G. et al. Carotid atherosclerosis and risk of subsequent coronary event in outpatients with atherothrombosis. Stroke 44(2), 373-379 (2013).

4. Hamrah, M. S. et al. Impact of airflow limitation on carotid atherosclerosis in coronary artery disease patients. Respiration 89(4), 322-328 (2015). 
5. Song, P. et al. Global and regional prevalence, burden, and risk factors for carotid atherosclerosis: A systematic review, metaanalysis, and modelling study. Lancet. Glob. Health. 8(5), e721-e729 (2020).

6. Clarke, R. et al. Burden of carotid artery atherosclerosis in Chinese adults: Implications for future risk of cardiovascular diseases. Eur. J. Prev. Cardiol. 24(6), 647-656 (2017).

7. Baber, U. et al. Prevalence, impact, and predictive value of detecting subclinical coronary and carotid atherosclerosis in asymptomatic adults: The BioImage study. J. Am. Coll. Cardiol. 65(11), 1065-1074 (2015).

8. Byrnes, K. R. \& Ross, C. B. The current role of carotid duplex ultrasonography in the management of carotid atherosclerosis: foundations and advances. Int. J. Vasc. Med. 2012, 187872 (2012).

9. Brott, T. G., et al. 2011 ASA/ACCF/AHA/AANN/AANS/ACR/ASNR/CNS/SAIP/SCAI/SIR/SNIS/SVM/SVS guideline on the management of patients with extracranial carotid and vertebral artery disease: executive summary: a report of the American College of Cardiology Foundation/American Heart Association Task Force on Practice Guidelines, and the American Stroke Association, American Association of Neuroscience Nurses, American Association of Neurological Surgeons, American College of Radiology, American Society of Neuroradiology, Congress of Neurological Surgeons, Society of Atherosclerosis Imaging and Prevention, Society for Cardiovascular Angiography and Interventions, Society of Interventional Radiology, Society of NeuroInterventional Surgery, Society for Vascular Medicine, and Society for Vascular Surgery. Developed in collaboration with the American Academy of Neurology and Society of Cardiovascular Computed Tomography. Catheter Cardiovasc Interv. 81(1), E76-123 (2013).

10. Jonas, D. E. et al. Screening for asymptomatic carotid artery stenosis: a systematic review and meta-analysis for the U.S. Preventive Services Task Force. Ann. Intern. Med. 161(5), 336-346 (2014).

11. Goldstein, L. B. et al. Guidelines for the primary prevention of stroke: a guideline for healthcare professionals from the American Heart Association/American Stroke Association. Stroke 42(2), 17-84 (2011).

12. Rajkomar, A., Dean, J. \& Kohane, I. Machine learning in medicine. N. Engl. J. Med. 380(14), 1347-1358 (2019).

13. Hu, X. et al. Machine learning to predict rapid progression of carotid atherosclerosis in patients with impaired glucose tolerance. EURASIP J. Bioinform. Syst. Biol. 2016(1), 14 (2016).

14. Li, X., Bian, D., Yu, J., Li, M. \& Zhao, D. Using machine learning models to improve stroke risk level classification methods of China national stroke screening. BMC Med. Inform. Decis. Mak. 19(1), 261 (2019).

15. Verma, L., Srivastava, S. \& Negi, P. C. A hybrid data mining model to predict coronary artery disease cases using non-invasive clinical data. J. Med. Syst. 40(7), 178 (2016).

16. Li, F. et al. Computer-assisted lip diagnosis on traditional Chinese medicine using multi-class support vector machines. BMC Compl. Altern Med. 12, 127 (2012).

17. Ogorodnyk O, Lyngstad OV, Larsen M, Wang K, Martinsen K. International Workshop of Advanced Manufacturing and Automation. Springer; Singapore: 2019. Application of machine learning methods for prediction of parts quality in thermoplastics injection molding, 237-244.

18. Carvalho, E. D. et al. Breast cancer diagnosis from histopathological images using textural features and CBIR. Artif. Intell. Med. 105, $101845(2020)$.

19. Ke, K. C. \& Huang, M. S. Quality prediction for injection molding by using a multilayer perceptron neural network. Polymers (Basel). 12(8), $1812(2020)$

20. Xia, J. et al. A model using support vector machines recursive feature elimination (SVM-RFE) algorithm to classify whether COPD patients have been continuously managed according to GOLD guidelines. Int. J. Chron. Obstruct. Pulmon. Dis. 15, 2779-2786 (2020).

21. Russell, S. \& Norvig, P. Artificial Intelligence: A Modern Approach 3rd edn. (Prentice Hall Press, 2009).

22. Zhangm A., Lipton, Z. C., Li, M., Smola, A. J. Dive into deep learning (release 0.7). Textbook for STAT 157 Introduction to Deep Learning at UC Berkeley, Spring 2019, 131-139.

23. Yu, W., Liu, T., Valdez, R., Gwinn, M. \& Khoury, M. J. Application of support vector machine modeling for prediction of common diseases: the case of diabetes and pre-diabetes. BMC Med. Inform. DecisMak 10, 16 (2010).

24. Chen T, He T, Benesty M, Khotilovich V, Tang Y. Xgboost: extreme gradient boosting. R package version 0.4-2. 2005; pp. 1-4

25. Friedman, J. H. Greedy function approximation: A gradient boosting machine. Ann. Stat. 29, 1189-1232 (2001).

26. Friedman, J., Hastie, T. \& Tibshirani, R. Additive logistic regression: A statistical view of boosting. Ann. Stat. 28, 337-407 (2000).

27. Breiman, L. Random forests. Mach. Learn. 45, 5-32 (2001).

28. Fine-Edelstein, J. S. et al. Precursors of extracranial carotid atherosclerosis in the Framingham Study. Neurology 44, 1046-1050 (1994).

29. van der Meer, I. M. et al. Risk factors for progression of atherosclerosis measured at multiple sites in the arterial tree: The Rotterdam Study. Stroke 34(10), 2374-2379 (2003).

30. Zhang, J. et al. Comparison of the characteristics and risk factors of carotid atherosclerosis in high stroke risk populations between urban and rural areas in North China. Front. Neurol. 11, 554778 (2020).

31. Azarpazhooh, M. R., Andalibi, M. S. S., Hackam, D. G. \& Spence, J. D. Interaction of smoking, hyperhomocysteinemia, and metabolic syndrome with carotid atherosclerosis: A cross-sectional study in 972 non-diabetic patients. Nutrition 79-80, 110874 (2020).

32. Chinese ultersound doctors association. Guidelines for vascular ultrasound examination. Chin. J. Ultrasonogr. 11, 993-1012 (2009).

33. Magnussen, C. G. et al. Use of B-mode ultrasound to examine preclinical markers of atherosclerosis: Image quality may bias associations between adiposity and measures of vascular structure and function. J. Ultrasound Med. 30(3), 363-369 (2011).

34. Chalasani, N. et al. The diagnosis and management of non-alcoholic fatty liver disease: practice guideline by the American Gastroenterological Association, American Association for the Study of Liver Diseases, and American College of Gastroenterology. Gastroenterology 142(7), 1592-1609 (2012).

\section{Acknowledgements}

This work was supported by the Guilin scientific research and technology development project (20190218-51) and Guangxi scientific research and technology development project (GuiKe AD16450020) in China.

\section{Author contributions}

Y.J., Y.Z. and S.C. designed the study. Q.Y., X.L., L.H. and P.Y. collected the data. S.C. and Y.J. developed the models and wrote the manuscript. All authors contributed to the revision and approved the final manuscript.

\section{Competing interests}

The authors declare no competing interests.

\section{Additional information}

Supplementary Information The online version contains supplementary material available at https://doi.org/ 10.1038/s41598-021-01456-3. 
Correspondence and requests for materials should be addressed to S.C.

Reprints and permissions information is available at www.nature.com/reprints.

Publisher's note Springer Nature remains neutral with regard to jurisdictional claims in published maps and institutional affiliations.

(c) (i) Open Access This article is licensed under a Creative Commons Attribution 4.0 International cc) License, which permits use, sharing, adaptation, distribution and reproduction in any medium or format, as long as you give appropriate credit to the original author(s) and the source, provide a link to the Creative Commons licence, and indicate if changes were made. The images or other third party material in this article are included in the article's Creative Commons licence, unless indicated otherwise in a credit line to the material. If material is not included in the article's Creative Commons licence and your intended use is not permitted by statutory regulation or exceeds the permitted use, you will need to obtain permission directly from the copyright holder. To view a copy of this licence, visit http://creativecommons.org/licenses/by/4.0/.

(C) The Author(s) 2021 